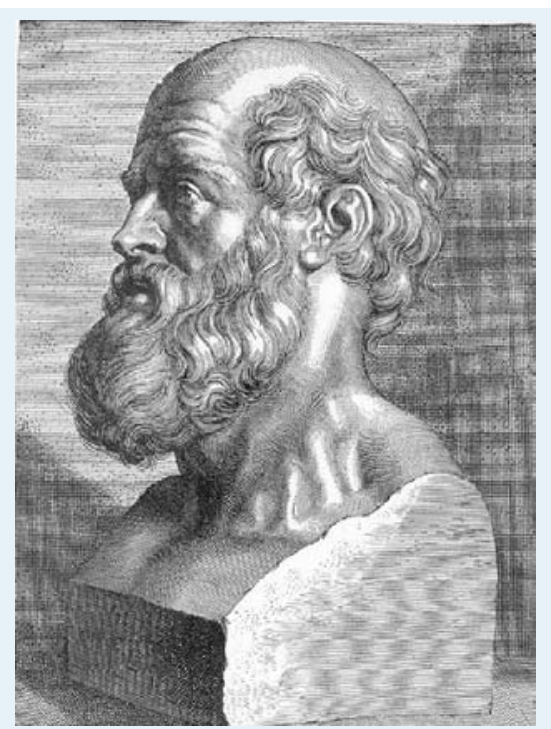

\section{Ernährung und Nahrungsergänzung}

«Lasst das Essen die Medizin sein», meinte vor knapp 2500 Jahren Hippokrates. Wie Vertreter der verschiedenen Fachrichtungen der Komplementärmedizin das Essen in ihre Therapie einbauen, erfahren Sie im Expertengespräch auf Seite 138. Und weil das, was Mutter Natur in unsere Lebensmittel hineingetan hat, schon lange nicht mehr reicht, um die Bedürfnisse des modernen Menschen zu befriedigen, berichten wir im nachfolgenden Beitrag über die Grundlagen und Anwendungen von Functional Foods. Diese reichen von Präbiotika bis hin zu cholesterolarmen Eiern. Dass Obst und Gemüse gesund erhalten, steht auf Seite 142. Mit jeder täglichen Portion sinkt das Infarktrisiko um $10 \%$.

\title{
Beim Essen wird der Natur nachgeholfen
}

Ergänzungsstoffe in der Nahrung und Functional Foods sind auf fast jedem Teller. Sie sollen Mangelerscheinungen verhindern, die Gesundheit fördern und Krankheiten vermeiden. Teilweise werden die Heilsversprechen der Hersteller in Studien belegt.

Gesunde Ernährung treibt die Konsumentinnen und Konsumenten um. Die jüngste Ess-Trend-Studie von Coop Schweiz zeigt, dass die Schweizerinnen und Schweizer von ihrem Essen erwarten, dass es gesund ist. Dabei soll es nicht einfach die Bedürfnisse des Körpers nach Energie, Kohlenhydraten, Vitaminen und Spurenelementen stillen. Gefragt ist eine Ernährung, die das Wohlbefinden steigert, die Gesundheit insgesamt verbessert und hilft, Krankheiten $\mathrm{zu}$ vermeiden. Längst hat sich die Nahrungsmittelindustrie auf dieses Bedürfnis eingestellt. Kaum noch ein Lebensmittel kommt neu auf den Markt, in dem nicht Inhaltsstoffe für Wohlbefinden oder Prävention sorgen sollen, wie z.B. Joghurt, in dem sich optimierte Mikroorganismen tummeln, die gut für die Verdauung sind, oder Milchdrinks, in denen sie das Abwehrsystem anregen sollen. Weltweit, so schätzen Experten, werden pro Jahr 2013 Milliarden Franken mit diesen Functional Foods umgesetzt. Die Nachfrage wächst rasant: sie nimmt pro Jahr um 7,4\% zu; am meisten steigt der Konsum von Getränken mit 10,8\%, während der der «festen» Lebensmittel nur um $6,9 \%$ steigt. Vor allem grosse, internationale Konzerne bestimmen das Angebot: Die Schweizer Nestlé, die französische DanoneGruppe oder der Lebensmittelriese Unilever beherrschen den Markt und legen den Konsumenten das Essen auf den Tisch, das sie brauchen, um ein ganzheitlich gesundes Leben zu führen. Neben den Functional Foods boomen die Ergänzungsstoffe. Egal ob es Vitamine sind, die als Antioxidantien dienen oder Mangelerscheinungen vermeiden sollen, oder z.B. Spurenelemente, sie werden als Pillen geschluckt, als Brausetabletten sprudelnd in Wasser aufgelöst oder in den Kochtopf gerührt. Der Markt wächst um jährlich 3,8\%. Vor allem kleinere Unternehmen bieten Produkte an, die in Drogerien und Apotheken an die Konsumentinnen und Konsumenten gebracht werden. Sie sollen als Vitalstoffe den Körper unterstützen sowie Eisenmangel oder ein Vitamindefizit beheben.

\section{Mehr als nur sättigen}

Eine ganzheitliche Ernährung soll nicht nur den Hunger stillen und die Körperfunktionen aufrechterhalten. Schon das vor über 100 Jahren entwickelte Bircher-Müsli mit seiner Mischung aus Früchten und Getreide sollte den Körper stärken, die Abwehrkräfte aufbauen und Krankheiten fernhalten. In den 1980er-Jahren wurde dafür in Japan erstmals die Bezeichnung Functional Food gebraucht. Seitdem tauchen Präbiotika und Probiotika, Ergänzungsstoffe und Ersatzstoffe in fast allen Sorten von Lebensmitteln auf.

Präbiotika sind nicht verdauliche Bestandteile der Nahrung, die die Vermehrung oder die Aktivität von Darmbakterien anregen. Die wichtigsten unter ihnen sind Fructo-Oligosaccharide, Inulin, Isomalto-Oligosaccharide, Polydextrose, Lactulose und Stärke. Weltweit werden davon jährlich 167000 Tonnen im Wert von 390 Millionen Euro verkauft.

\section{KARGER}

Fax +497614520714 Information@Karger.d www.karger.com 
Oligosaccharide werden hauptsächlich bei der Behandlung von Übergewicht eingesetzt, weil sie ein Sättigungsgefühl auslösen und so den Hunger reduzieren, wie eine Reihe von Studien belegt. Inulin und Oligofructose erhöhen die Aufnahmefähigkeit des Körpers für Kalzium und verbessern dadurch die Mineralienzusammensetzung und die Dichte der Knochen. Ausserdem fördern sie die Bildung von Glucose im Blut und senken den Spiegel von Cholesterol und Serumlipiden. Für Probiotika schaffen die Präbiotika bessere Lebensbedingungen: deren Vermehrung wird dadurch angeregt. Diesen Effekt machen sich Hersteller von Functional Food zunutze und vermarkten Pro- dukte als Synbiotika, die Prä- und Probiotika enthalten.

\section{Biotech in Milchprodukten \\ hat lange Tradition}

Unter Probiotika (Tab. 1) versteht man Mikroorganismen, die einen positiven Effekt auf die Gesundheit ausüben. Probiotika finden sich vor allem in Milchprodukten, um sie zu Functional Foods zu machen. Egal ob Milchdrink oder Joghurt, sobald sich Mikroorganismen, denen eine Wirkung auf Wohlbefinden und Gesundheit zugeschrieben wird, darin befinden, werden sie zu Verkaufsschlagern. Sie machen zwei Drittel des europäischen Markts der Functional Foods aus. Den Weg an die Spitze der Be- liebtheit bei den Konsumentinnen und Konsumenten hat ihnen die traditionell grosse Bedeutung von Milch in der täglichen Ernährung in Europa geebnet. Es ist erstaunlich, dass beim allgemeinen Unbehagen gegenüber «Genfood» in der Schweiz gerade Lebensmittel so begehrt sind, die mit biotechnologischen Methoden hergestellt und deren Mikroorganismen auch verändert wurden. In einer grossen Studie (Acta Universitatis Latviensis, 2006, Vol. 710, Biology, pp. 117-129) über Bedeutung von Milch unter den Functional Foods kommen Malda Maija Toma und Juris Pokrotnieks von der Universität Riga zu dem Schluss, dass Functional Milk akzeptiert werde, weil fermentierte Milch-

Tab. 1. Die Einsatzfelder von Probiotika und die wahrscheinlichen Wirkmechanismen (Quelle: Sanders ME: Probiotics: considerations for human health. Nutr Rev 2003;61:91-99)

\begin{tabular}{|c|c|c|}
\hline Ziel & Indikation & Wirkung \\
\hline \multirow[t]{2}{*}{ Verdauung } & $\begin{array}{l}\text { Irritable Bowel Syndrome, allgemeine Verdauungsbe- } \\
\text { schwerden (Verstopfung, nichtpathologische Diarrhö, } \\
\text { Distension, Flatulenz, Krämpfe, Mundgeruch, der } \\
\text { durch Verdauungsbeschwerden hervorgerufen wird) }\end{array}$ & $\begin{array}{l}\text { Veränderungen der Populationsgrösse oder der Aktivität der } \\
\text { Darmflora }\end{array}$ \\
\hline & Laktoseintoleranz & Mikrobielle Laktase wird im Dünndarm zur Verfügung gestellt \\
\hline \multirow[t]{9}{*}{ Abwehrsystem } & $\begin{array}{l}\text { Allergien wie atopische Ekzeme, Milchallergie, } \\
\text { rheumatoide Arthritis }\end{array}$ & Translokation, Barriereeffekte \\
\hline & Karies & $\begin{array}{l}\text { Veränderungen in der Populationsgrösse und der Aktivität der } \\
\text { oralen Mikroflora und ihrer Fähigkeit, sich an die Zähne anzu- } \\
\text { haften }\end{array}$ \\
\hline & $\begin{array}{l}\text { Krebserregende Stoffe, mutagene mikrobielle Aus- } \\
\text { scheidungen }\end{array}$ & $\begin{array}{l}\text { Absorption des Mutagens, Stimulation des Immunsystems, } \\
\text { Inhibition der Karzinogenproduktion in der Darmflora }\end{array}$ \\
\hline & $\begin{array}{l}\text { Diarrhö, die durch Antibiotika oder Rotavirus her- } \\
\text { vorgerufen wird, Colitis durch Clostridium difficile, } \\
\text { nosokomiale Diarrhö }\end{array}$ & $\begin{array}{l}\text { Kompetitive Verdrängung, Translokation, Barriereeffekt, } \\
\text { verstärkte Immunreaktion }\end{array}$ \\
\hline & Helicobacter pylori & Antipathogene Aktivität \\
\hline & Immunmodulation, Immunstatus, Immunreaktion & $\begin{array}{l}\text { Interaktion mit den Immunzellen oder Zellrezeptoren, die zu } \\
\text { einer Zunahme in der Phagozytoseaktivität der weissen Blut- } \\
\text { körperchen führt; Zunahme des IgA-Spiegels nach der Antigen- } \\
\text { exposition, Zunahme der Proliferation der intraepithelialen } \\
\text { Leukozyten, Regulation des Th1-/Th2-Verhältnisses, Induktion } \\
\text { der Zytokinsynthese }\end{array}$ \\
\hline & $\begin{array}{l}\text { Intestinale Entzündungen, Colitis ulcerosa, } \\
\text { Morbus Crohn, Pouchitis }\end{array}$ & Reduktion der Immunantwort \\
\hline & Starkes Wachstum der Darmbakterien & Antimikrobielle Aktivität, kompetitive Verdrängung \\
\hline & Bakterielle Vaginosis, Infektionen der Harnwege & Antimikrobielle Aktivität, kompetitive Verdrängung \\
\hline \multirow[t]{4}{*}{ Andere } & Senkung des Blutcholesterols & Dekonjugation der Gallensäure \\
\hline & Endotoxämie mit Zirrhose & Inhibition der Produktion von Endotoxinen durch die Darmflora \\
\hline & Hypertension & $\begin{array}{l}\text { Zellbausteine oder Peptide, die aus der Fermentation stammen, } \\
\text { wirken als ACE-Inhibitoren }\end{array}$ \\
\hline & Nierensteine & $\begin{array}{l}\text { Veränderungen in der Mikroflora, die den Abbau von Oxalaten } \\
\text { beeinflussen }\end{array}$ \\
\hline
\end{tabular}




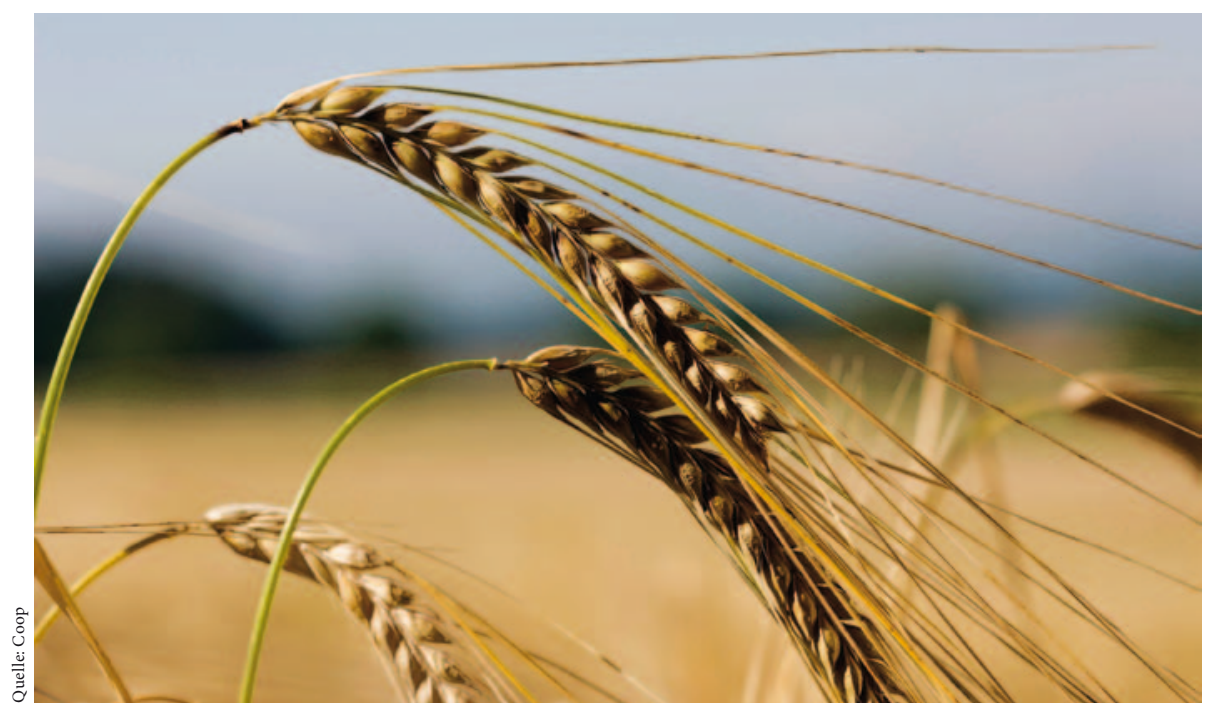

produkte und deren biotechnologische Herstellungsmethoden seit Menschengedenken verwendet werden. Seit mehr als 6000 Jahren stehen sie hier auch bei Erwachsenen auf dem Speiseplan. Schon der römische Historiker Plinius empfahl vor fast 2000 Jahren gegorene Milch, um Erkrankungen des Magen-Darm-Traktes zu behandeln und Infektionen auf den Leib zu rücken. Anfang des 20. Jahrhunderts empfahl der französische Kinderarzt Henry Tissier den Einsatz von Bifidobakterien zur Prävention von Krankheiten in den ersten beiden Lebensjahren. Die Mikroorganismen sollen helfen, Infektionen zu verhindern. Bifidobakterien finden sich in grosser Menge in der Darmflora von Säuglingen, die gestillt werden. Der russische Bakteriologe und Nobelpreisträger Ilja Metschnikov war überzeugt, dass das Trinken von fermentierter Milch die zersetzende Wirkung der Darmflora auf die Darmwand vermindern könne. Mit der Wirkung der Probiotika im Intestinaltrakt hat sich die Forschung in den letzten 20 Jahren intensiv beschäftigt. In placebokontrollierten Studien konnte die gesundheitsfördernde Wirkung verschiedener Bakterienstämme nachgewiesen werden. Sie scheinen einen beruhigenden Einfluss auf die Mucosaschicht auszuüben und das Immunsystem zu stärken. In verschiedenen Untersuchungen konnten Wissenschafter auch Belege dafür finden, und Fructo-Oligosaccharide, und Stärke, die als Präbiotika dienen. Daneben sind sie auch Ballaststoffe und sollen positiv auf die Physiologie wirken. Bestandteile von Getreide, wie Beta-Glucan, werden heute in der Lebensmittelindustrie verwendet, um fettarme Glace und Joghurt herzustellen. Sie sorgen dafür, dass sie genauso schmecken wie die fettreichen herkömmlichen Produkte. Der Stoff scheint Zukunftspotenzial zu haben. Die EU fördert die Forschung über den Einsatz von verbesserten Beta-Glucanen in der Lebensmittelherstellung.

Backwaren sind schon seit längerer Zeit zu Functional Food geworden, weil sie ideal sind, um zusätzliche Inhaltsstoffe zu transportieren: Der Lebensmittelmulti Unilever hat etwa ein Weissbrot auf den Markt gebracht, das Nährstoffe enthält, die sonst nur in dunklem Brot $\mathrm{zu}$ finden sind: Fasern, die Vitamine B1, B3 und B6 sowie Eisen, Zink oder Inulin. haltsstoffen angereichert sind. Etwa $1 \%$ der z.B. in Deutschland getrunkenen Säfte sind solche Vitaminsäfte. Daneben sind solche auf dem Markt, die die Cholesterolwerte senken sollen. Sie enthalten Sojabestandteile und Omega-3-Fettsäuren, den derzeit am meisten beworbenen Zusatz zu Functional Foods. In den Regalen stehen auch Säfte, die die Sehkraft fördern sollen, weil sie Lutein enthalten, oder solche, die gut für die Knochen sein sollen, weil sie Kalzium und Inulin enthalten. Angereicherte Säfte, die Inulin, L-Carnitine, Vitamine, Kalzium und Magnesium als Wirkstoffe enthalten, sind ebenfalls im Angebot. Vor sechs Jahren lag der Marktanteil dieser «Functional Drinks» in Europa bei $7 \%$, heute liegt er bei knapp 10\%: Im letzten Jahr wurden in Europa mehr als fünf Milliarden Liter davon getrunken.

Functional Foods aus Getreide sind entweder mit bestimmten Stoffen angereichert oder sollen die Vermehrung von Probiotika fördern, wie etwa von Lactobacilli und Bifidobakterien im Darm. Sie enthalten wasserlösliche Fasern, wie Beta-Glucan und Arabinoxylan, Oligosaccharide, wie Galacto-

\section{Nach der Margarine}

kommt die fettarme Butter

Brotaufstriche, die den Cholesterolspiegel senken, sind seit Langem auf dem Markt. Eine Butter mit niedrigem Cholesterol gibt es seit fast 20 Jahren in den Kühlregalen in Belgien. Bei ihr sind $90 \%$ des Cholesterols im Milchfett durch die Zugabe von BetaCyclodextrin ersetzt. Durch diesen Stoff werden auch andere Milchprodukte wie Käse oder Sahne cholesterolarm gemacht. Verschiedene Margarinesorten enthalten seit langem Phytosterole und -stanole, die den Cholesterinspiegel senken sollen. Die britische Freshlay Foods hat die VITA Eggs auf den Markt gebracht. Diese Eier sind mit Omega-3-Fettsäuren, Antioxidantien und anderen Vitaminen angereichert. Schon seit über 10 Jahren sind ähnliche Eier in verschiedenen Ländern in Europa auf dem Markt. Vom Ei «Columbus», das künstlich mehr Vitamin E und Omega-3-Fettsäuren enthält, werden jedes Jahr mehr als 50 Millionen Stück gelegt. (rfi) 